\title{
Viscoelastic Behaviour of Maturating Green Poplar Wood
}

\author{
Guillaume Pot, Evelyne Toussaint, Catherine Coutand, and Jean-Benoît Le Cam
}

\begin{abstract}
Trees can modify the orientation of their trunk and branches by asymmetrical production of reaction wood. Even though biomechanical models that simulate gravitropic movements are proposed in the literature, recent studies show that they cannot fit the righting-up movement of young, fast growing poplar trees at the intra annual scale. This discrepancy could be explained by the fact that cell maturation is not considered in these models. Another important point is that the knowledge of viscoelastic properties of green wood is poorly studied in the literature, while some biomechanical models show that viscoelasticity of wood can be an important parameter in the tree gravitropic response. These models only provide qualitative results and the viscoelastic properties of wood have to be characterized. The goal of this work is to study the evolution of viscoelastic properties of green wood during maturation. The effects of viscoelasticity corresponding to mechanical solicitation (stress or strain) can be studied by creep or relaxation tests. For the wood of standing tree, internal stresses are due to the maturation and growth of the tree. These constraints can thus act as mechanical stresses and cause deformation of green wood specimens. The effect of these internal stresses on specimens taken from the trunk is first studied in the present work. In the second part of the paper, a study of creep bending of clamped-free wooden boards cut within the last growth ring is presented.
\end{abstract}

Keywords Green wood $\bullet$ Viscoelasticity $\bullet$ Maturation $・$ Burgers' model $\bullet$ Internal maturation stresses

\subsection{Introduction}

Gravitropic movements of trees allow them to control their posture by maintaining and modifying the orientation of their trunk and branches. These movements are ensured thanks to asymmetrical differentiation of reaction wood also called tension wood (TW) in most deciduous species and that is produced on one side of the axis [1]. Wood which is localized on the other side of the axis is called opposite wood (OW). The difference of internal stresses between the two sides of the axis enables the movements of the trunk. Wood cells are created at the periphery of the trunk between wood and bark [2], and during the maturation, wood cells tend to shrink in the longitudinal direction. These maturation strains are locked by the cells created earlier which are already stiffer. Consequently, internal maturation stresses are produced.

Several models that enable the internal maturation stresses to be determined can be found in the literature [3, 4]. More particularly, Dlouhà et al. [5] and Coutand et al. [6] have shown that viscoelasticity can increase the righting-up efficiency of trees. In order to determine quantitatively the effect of such behaviour of wood in the gravitropic process, experimental studies must be performed. Because wood contains a large amount of water and thus cells moisture content is above fiber

G. Pot $\bullet$ E. Toussaint $(\bowtie)$

Clermont Université, Université Blaise Pascal, Institut Pascal, BP 10448, F-63000 Clermont-Ferrand, France

CNRS, UMR 6602, Institut Pascal, F-63171 Aubiere, France

e-mail: evelyne.toussaint@univ-bpclermont.fr

C. Coutand

Université Blaise Pascal, UMR 547 PIAF, INRA, Clermont Université, 63100 Clermont-Ferrand, France

J.-B. Le Cam

Université de Rennes 1, LARMAUR - ERL CNRS 6274, Campus de Beaulieu, Bât. 10B, 35042 Rennes Cedex, France 
saturation point, most of the studies that deal with wood viscoelastic properties focus on mechano-sorptive behavior [7]. In the frame of the modeling of tree gravitropism, the viscoelastic properties of green wood, i.e. wood above fiber saturation point are necessary. Only few studies are dedicated to the experimental characterization of the viscoelastic behaviour of green wood (see Dlouhà et al. [8], Kojima and Yamamoto [9] for instance). None of them has studied the viscoelastic behaviour of TW, or the effect of maturation on viscoelastic properties.

The aim of the present work is to characterize the viscoelastic properties of green poplar wood in order to enhance the prediction of biomechanical models of the gravitropic process. The effect of the maturation process on viscoelasticity is also discussed. An experimental methodology is proposed in order to eliminate the effect of internal maturation stresses in the time-dependent behaviour of wood samples. The materials and methods used are first described. Then, results are presented and discussed.

\subsection{Materials and Methods}

\subsubsection{Experimental Set-up}

Seven two-years-old hybrid poplars (Populus deltoides x Populus nigra, cv I4551) planted in individual 60 L containers were tilted at about $35^{\circ}$ from the vertical at the beginning of the third year of growth. Consequently, TW was produced on the upper-side of the tilted axis (see Fig. 54.1a). The diameter growth rate in the basal part of the tree (lower than $20 \mathrm{~cm}$ from ground) was calculated using weekly trunk diameter measurements. It was about $0.16 \pm 0.03 \mathrm{~mm} / \mathrm{day}$. One tree was felled approximately each week during 2 months. Thus, a felling time after tilting can be defined. A piece of the trunk of $8 \mathrm{~cm}$ in length was taken from the basal part of each felled tree. A board $6 \mathrm{~mm}$ in width in the tangential direction was cut using a band saw (see Fig. 54.1a). Then, after the bark was manually removed, approximately $1.3 \mathrm{~mm}$ thick slats were cut using a razorblade (see Fig. 54.1b). Cutting with a razorblade induces no loss of material, so the complete sequence of slats represents the last intra-ring radial variation of wood. Slats were set in water in plastic containers once they were cut in order to keep wood above fiber saturation point. They were left at room temperature (about $20^{\circ} \mathrm{C}$ ). These slats were directly used for curvature measurements and creep tests.

\subsubsection{Determination of Wood Cells Age}

Wood cells age was determined under the assumption of a constant growth rate on both sides of the trunk. For that purpose, the eccentricity $\delta$ of the trunk and the radial position of each slat were measured. Then, by plotting $\delta$ versus the difference between the radius of the tree at a given time $r$ and the radius at the beginning of the growing season $r_{0}$, an eccentricity coefficient $\mathrm{K}_{\mathrm{d}}$ equal to 0.4 was determined. Finally, thanks to experimental values, growth rates of upper side $\mathrm{G}_{\mathrm{tw}}$ and of lower side $G_{\text {ow }}$ were about $G_{\mathrm{tw}}=0.12 \mathrm{~mm} /$ day and $G_{\mathrm{ow}}=0.050 \mathrm{~mm} /$ day. Finally, this method enables properties to be represented according to the wood cells age, therefore to different maturation times.

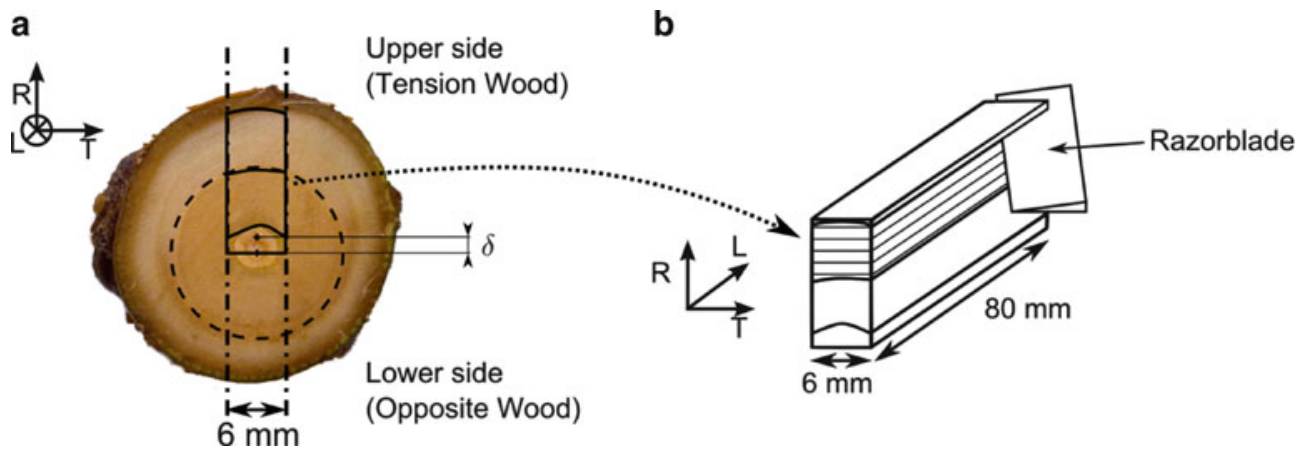

Fig. 54.1 Preparation of the samples. (a) Image of a cross-section of the trunk (b) drawing of the half board 

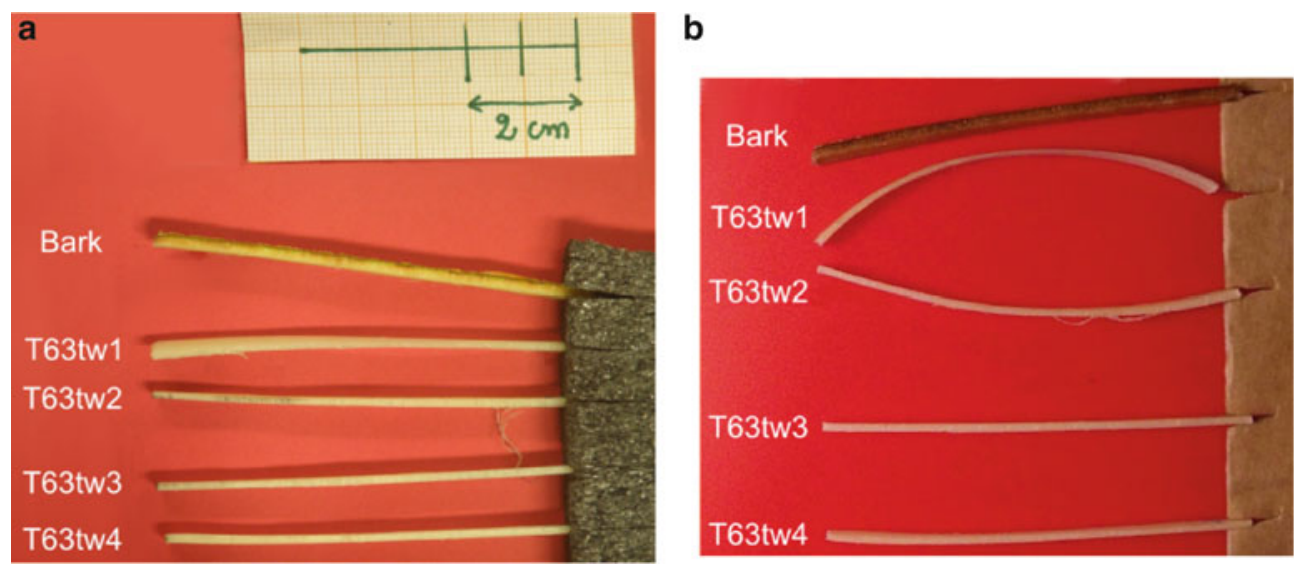

Fig. 54.2 TW samples of tree T63 in the longitudinal-radial plane: (a) 5 min after cutting, (b) 1 week after cutting

\subsubsection{Autonomous Curvature Measurements}

In the tree, internal maturation stresses exists in the three directions but are the highest in the longitudinal direction [2]. Sawing operations allow to release these stresses. Moreover, within the thickness of each slat, maturation of wood cells induces a gradient of properties. Finally, when slats are sawed, a curvature in the longitudinal-radial plane can occur.

Curvatures of hydrated slats were experimentally determined using full field measurements. For that purpose, high definition images $\left(3648 \times 2736\right.$ pixels $\left.^{2}\right)$ of the samples were first recorded immediately after cutting. Then, images were regularly taken for 8 weeks (see Fig. 54.2). The autonomous curvature of some samples increased with time. The shape of each slat was fitted by a circle using the least squares method with a MATLAB routine [10]. Curvature was defined as positive when center of curvature was on the side towards the center of the trunk, and defined as negative otherwise. The coefficient of correlation was above 0.95 for all the samples. Finally, a curvature radius was attributed to each image of each sample during slat autonomous deformation.

\subsubsection{Creep Tests}

After internal maturation stresses of the previous samples were fully relaxed, bending creep test were performed in a water saturated media (see Fig. 54.3). The samples were anchored at one end by clamping them over a length of $22 \mathrm{~mm}$ between two aluminium plates maintained by a screw. Weights were suspended from the free end of the samples; thus a constant load was applied. The samples of trees T49 and T63 (that is trees felled after 49 and 63 days respectively) were tested just after autonomous curvature measurements.

Deflection of the samples was measured thanks to two vertical reference points chosen in scales submerged into the aquarium and located in the same plane as the samples (see Fig. 54.3). Deflection was calculated as the vertical difference between unloaded state and loaded state. The spatial resolution was about $0.1 \mathrm{~mm}$. The samples tested present different maturating states, and consequently different stiffnesses. The applied load was calculated for each slat, using experimental preliminary results obtained on three-point bending tests [11] and a theoretical initial deflection of $3 \mathrm{~mm}$. As a first approximation, the compliance $\mathrm{J}(\mathrm{t})$ was calculated using the following equation

$$
J(t)=\frac{3 I v(t)}{P L^{3}}
$$

where $\mathrm{I}$ is the second moment of area, $\mathrm{v}(\mathrm{t})$ is the deflection which depends on time, $\mathrm{P}$ is the applied load and $\mathrm{L}$ is the free sample length which mean value was $54 \pm 1 \mathrm{~mm}$. 
Fig. 54.3 Experimental set-up for creep tests

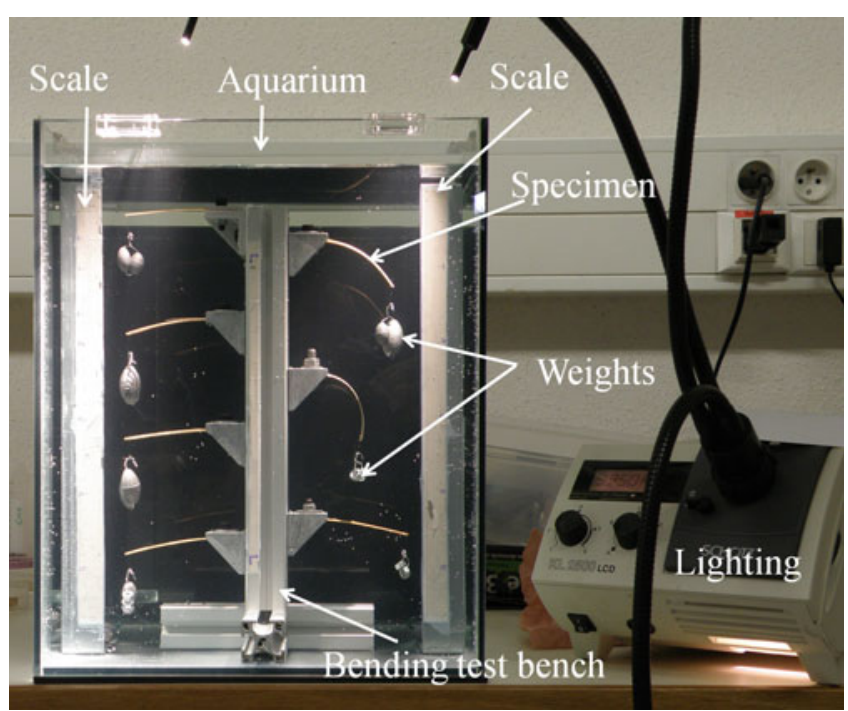

\subsection{Results and Discussion}

\subsubsection{Behaviour of Small Slats Under Internal Maturation Stresses}

Figure 54.4 shows the representative temporal evolution of the autonomous curvature of samples cut from the same tree and subjected to their internal maturation stresses. Initial curvature is closed to zero. Autonomous curvature increases significantly in absolute value for TW samples close to the cambium (see for example samples number 1 and 2). Neither the other TW sample in the inner part of the ring nor OW samples display curvature.

Autonomous curvatures are principally due to a gradient of internal maturation stresses from the external side to the internal side of the slat. This stress gradient produces a bending moment that induces an autonomous curvature if the samples are thin enough to flex. Since green wood is a viscoelastic material, the curvature changes with time as creep occurs. At the same time, stresses progressively relax. TW samples close to the cambium present a positive curvature while second TW samples present a negative curvature. In the literature, tree internal maturation stresses are calculated under the assumption of instantaneous maturation and elastic behaviour of wood [2-4]. The result is an internal maturation stress logarithmic curve that exhibits a radial gradient of stresses in the trunk. It is represented by a dashed line in Fig. 54.5.

Since it is monotonous, this stress profile cannot explain the different autonomous curvature directions between first and second TW samples. Actually, if we consider that cells close to the cambium are not totally mature, then there is a positive gradient of stresses while the cells are maturating. Furthermore, the maturation of peripheral cells induces compression of the inner cells, therefore the stress gradient becomes negative for this inner cells (similarly to the instantaneous maturation models of the literature). Figure 54.5 shows such a supposed stress profile. This stress profile can explain the difference in sign of autonomous curvature of the slats.

\subsubsection{Creep Tests Results}

Creep curves of tree T63 are presented in Fig. 54.6. Both TW and OW creep curves first present an exponential part, then reach an oblique asymptote. This behaviour can be modeled by Burgers' model. The creep function of this model is given by the following function:

$$
J(t)=\frac{1}{E_{0}}+\frac{t}{\eta_{\infty}}+\frac{1}{E_{1}}\left(1-e^{-t / \tau}\right)
$$


Fig. 54.4 Typical results in terms of temporal evolution of samples' autonomous curvature (tree T63)
Fig. 54.5 Diagram of probable internal maturation stress profile on TW side
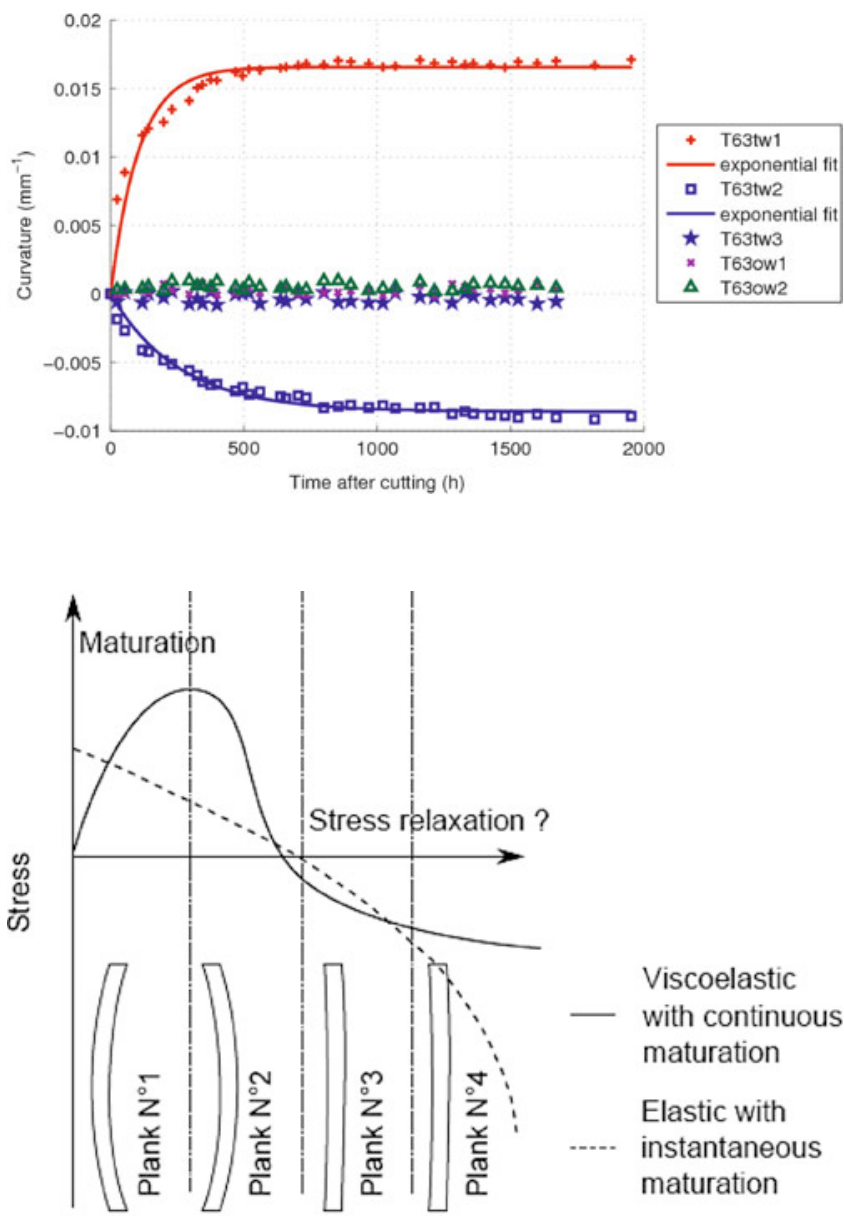

Radial distance from the cambium

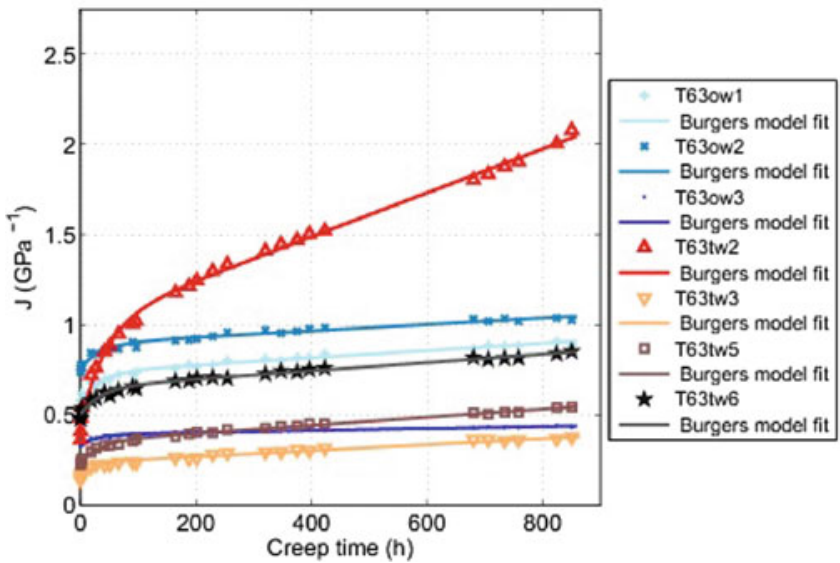

Fig. 54.6 Compliance according to creep time for tree T63 where $E_{0}$ is the elastic modulus, $\eta_{\propto}$ is the linear viscosity of the dashpot in series, $E_{1}$ is the spring modulus in parallel and $\tau=\frac{\eta_{1}}{E_{1}}$ is the relaxation time.

A numerical fit of the creep curves enables us to obtain the four viscoelastic parameters of Burgers' model. Under the assumption of a constant maturation process in terms of viscoelastic properties during the vegetative season, these results correspond to the evolution of the viscoelastic properties of green poplar wood according to maturation time (Fig. 54.7).

The variations with wood cells age of viscoelastic parameters appear very similar between OW and TW samples. This would seem to show that viscoelastic behaviour does not depend on the type of wood considered. The higher the maturation 
a

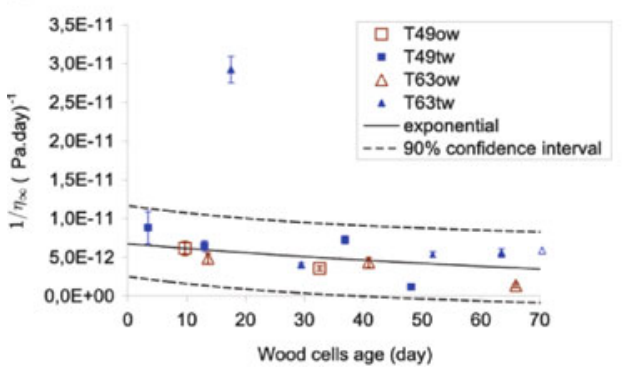

b

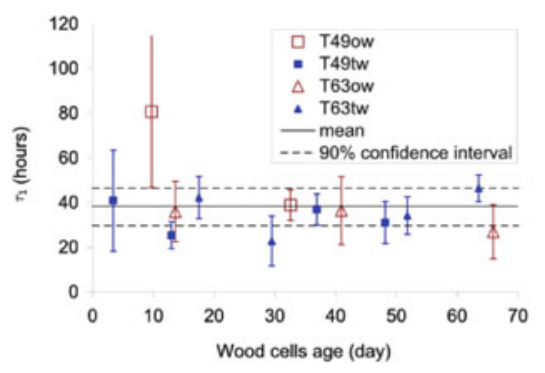

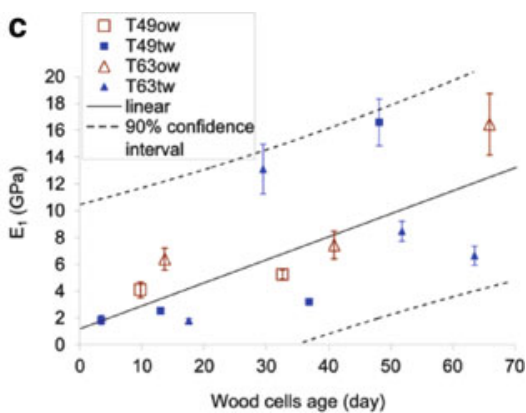

Fig. 54.7 Burgers' model parameters versus wood cell age for two different trees and both OW and TW

of wood, the higher E1 and $\eta_{\infty}$. So wood tends to lose its viscoelastic behaviour with maturation. $\tau$ is quasi constant and its mean value is $38 \pm 8 \mathrm{~h}$. Finally, the results present quite high variability that can be attributed to wood itself. They cannot be compared to any data in literature since no study dealing with poplar green wood viscoelasticity exists. Despite of their variability, these results allow us to obtain reasonable value of the viscoelastic parameters of green poplar wood.

\subsection{Conclusion}

In this study, the viscoelastic behaviour of small slats of green poplar wood has been studied. Samples were first subjected to their own, internal maturation stresses. Only the two first TW slats in the outer part of the trunk curve with time. These results can be explained by internal maturation stress relaxation. After autonomous curvature of the samples stopped and thus stresses were fully relaxed, creep test were performed on the same specimens. Creep curves of each samples have been fitted using a Burgers' rheological model and its viscoelastic parameters were determined according to the mean wood cells age of each sample. Green wood shows a tendency to lose its viscoelastic behaviour with maturation. The results of this experimental study provide information on the viscoelasticity of green wood that can be useful for other works dealing with green wood. In particular, the viscoelastic parameters obtained will be used in a biomechanical model such as Coutand et al. model's [6] in order to simulate gravitropic reaction with experimental values of viscoelastic parameters.

\section{References}

1. Scurfield G (1973) Reaction wood: its structure, function. Science 179:647-655

2. Fournier M, Chanson B, Thibaut B, Guitard D (1991) Mechanics of standing trees: modelling a growing structure submitted to continuous and fluctuating loads. 2. Tridimensional analysis of maturation stresses. Case of standard hardwood. Ann For Sci 48:527-546

3. Kubler H (1959) Studies on growth stresses in trees. 1. The origin of growth stresses and the stresses in transverse direction. Holz als Roh Werkstoff 17:1-9

4. Archer RR, Byrnes FE (1974) On the distribution of tree growth stresses. Part I: An anisotropic plane strain theory. Wood Sci Technol 8:184-196

5. Dlouhà J, Alméras T, Clair B et al (2008) Biomechanical performances of trees in the phase of active reorientation. Acta Univ Agric Silv Mendel Brun 56:39-44

6. Coutand C, Mathias J-D, Jeronimidis G, Destrebecq J-F (2011) TWIG: a model to simulate the gravitropic response of a tree axis in the frame of elasticity and viscoelasticity, at intra-annual time scale. J Theor Biol 273:115-129

7. Bowyer JL, Shmulsky R, Haygreen JG (2007) Forest products and wood science: an introduction, 5th Rev edn. Iowa State University Press, Ames

8. Dlouha J, Clair B, Arnould O et al (2009) On the time-temperature equivalency in green wood: characterisation of viscoelastic properties in longitudinal direction. Holzforschung 63:327-333

9. Kojima Y, Yamamoto H (2004) Effect of microfibril angle on the longitudinal tensile creep behavior of wood. J Wood Sci 50:301-306

10. Gilat A (2004) MATLAB: an introduction with applications, 2nd edn. Wiley, Hoboken

11. Pot G (2012) Mechanical characterization of green wood during maturation process and modeling of gravitropic reaction of young poplar. Thesis of Blaise Pascal University 